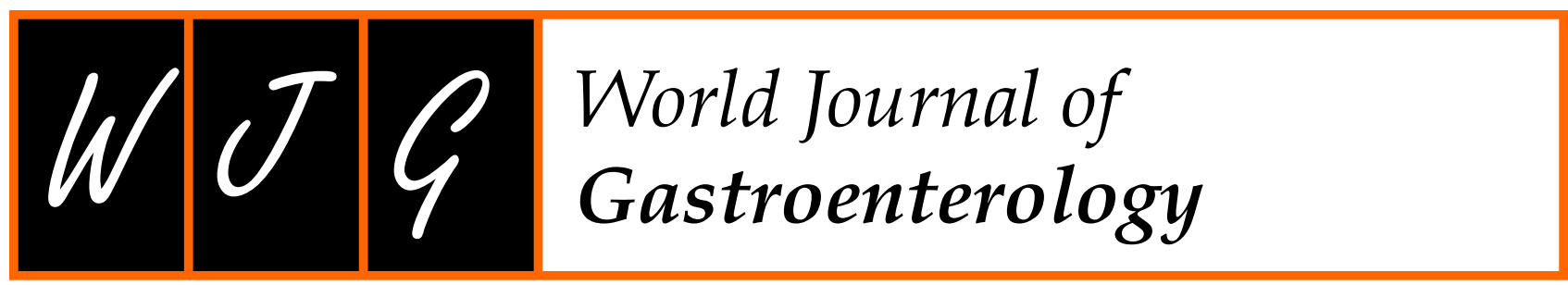

\title{
Validation of conventional non-invasive fibrosis scoring systems in patients with metabolic associated fatty liver disease
}

\author{
Yin-Lian Wu, Rahul Kumar, Ming-Fang Wang, Medha Singh, Jiao-Feng Huang, Yue-Yong Zhu, Su Lin
}

ORCID number: Yin-Lian Wu 00000001-9298-8367; Rahul Kumar 00000002-5092-4821; Ming-Fang Wang 0000-0001-7306-955X; Medha Singh 0000-0002-3258-0658; Jiao-Feng Huang 0000-0003-1383-6897; Yue-

Yong Zhu 0000-0002-0746-4911; Su Lin 0000-0001-7517-9859.

Author contributions: $\mathrm{Wu} \mathrm{YL}$, Kumar R and Lin S designed the study; Wang MF and Singh M analyzed the data; Huang JF collected the data; $\mathrm{Wu} Y \mathrm{~L}$ and Kumar R wrote the paper; Zhu YY and Lin $S$ supervised the study;

Wu YL and Kumar R contributed equally to this work; All authors contributed to the manuscript for important intellectual content and approved the submission.

Supported by Chinese National 13th Five-Year Plan's Science and Technology Projects, No. $2017 Z X 10202201$.

\section{Institutional review board} statement: The study protocol was approved by the Institutional

Review Board of the First Affiliated Hospital of Fujian Medical University and was in accordance with the Declaration of Helsinki.

Informed consent statement: All study participants, or their legal guardian, provided informed written consent prior to study
Yin-Lian Wu, Ming-Fang Wang, Jiao-Feng Huang, Yue-Yong Zhu, Su Lin, Department of Hepatology, Hepatology Research Institute, The First Affiliated Hospital, Fujian Medical University, Fuzhou 350005, Fujian Province, China

Rahul Kumar, Department of Gastroenterology and Hepatology, Duke-NUS academic Medical Centre, Changi General Hospital, Singapore 529889, Singapore

Medha Singh, Department of General Medicine, Tan Tock Seng Hospital, Singapore 308433, Singapore

Corresponding author: Su Lin, MD, PhD, Associate Chief Physician, Professor, Department of Hepatology, Hepatology Research Institute, The First Affiliated Hospital, Fujian Medical University, No. 20 Chazhong Road, Taijiang District, Fuzhou 350005, Fujian Province, China. sumer5129@fjmu.edu.cn

\section{Abstract \\ BACKGROUND}

Non-invasive fibrosis scores are not yet validated in the newly defined metabolic associated fatty liver disease (MAFLD).

\section{AIM}

To evaluate the diagnostic performance of four non-invasive scores including aspartate aminotransferase to platelet ratio index (APRI), fibrosis-4 index (FIB-4), body mass index, aspartate aminotransferase/alanine aminotransferase ratio, diabetes score (BARD), and nonalcoholic fatty liver disease fibrosis score (NFS) in patients with MAFLD.

\section{METHODS}

Consecutive patients with histologically confirmed MAFLD were included. The discrimination ability of different non-invasive scores was compared.

\section{RESULTS}

A total of 417 patients were included; 156 (37.4\%) of them had advanced fibrosis (Metavir $\geq$ F3). The area under receiver operating characteristic curve of FIB-4, NFS, APRI, and BARD for predicting advanced fibrosis was 0.736, 0.724, 0.671, and 0.609 , respectively. The area under receiver operating characteristic curve of FIB-4 and NFS was similar $(P=0.523)$, while the difference between FIB-4 and APRI $(P=0.001)$ and FIB-4 and BARD $(P<0.001)$ was statistically significant. The 
enrollment.

Conflict-of-interest statement: The authors have no conflict of interest to declare.

\section{Data sharing statement: Data are} available on request.

\section{Open-Access: This article is an} open-access article that was selected by an in-house editor and fully peer-reviewed by external reviewers. It is distributed in accordance with the Creative Commons Attribution NonCommercial (CC BY-NC 4.0) license, which permits others to distribute, remix, adapt, build upon this work non-commercially, and license their derivative works on different terms, provided the original work is properly cited and the use is non-commercial. See: htt p:/ / creativecommons.org/License s/by-nc/4.0/

Manuscript source: Unsolicited manuscript

Specialty type: Gastroenterology and hepatology

\section{Country/Territory of origin: China}

\section{Peer-review report's scientific} quality classification

\author{
Grade A (Excellent): A \\ Grade B (Very good): B \\ Grade C (Good): 0 \\ Grade D (Fair): 0 \\ Grade E (Poor): 0
}

Received: January 6, 2021 Peer-review started: January 6 , 2021

First decision: May 5, 2021

Revised: May 15, 2021

Accepted: July 29, 2021

Article in press: July 29, 2021

Published online: September 14, 2021

P-Reviewer: Sawada K

S-Editor: Gao CC

L-Editor: Filipodia

P-Editor: Liu JH best thresholds of FIB-4, NFS, APRI, and BARD for diagnosis of advanced fibrosis in MAFLD were 1.05, -2.1, 0.42, and 2. A subgroup analysis showed that FIB-4, APRI, and NFS performed worse in the pure MAFLD group than in the hepatitis $B$ virus-MAFLD group.

\section{CONCLUSION}

APRI and BARD scores do not perform well in MAFLD. The FIB-4 and NFS could be more useful, but a new threshold is needed. Novel non-invasive scoring systems for fibrosis are required for MAFLD.

Key Words: Metabolic associated fatty liver disease; Non-invasive fibrosis scores; Fibrosis-4 index; Non-alcoholic fatty liver disease fibrosis score; Aspartate aminotransferase to platelet ratio index; BARD

CThe Author(s) 2021. Published by Baishideng Publishing Group Inc. All rights reserved.

Core Tip: Metabolic associated fatty liver disease (MAFLD) is a new concept proposed in 2020 to redefine fatty liver disease. The utility of non-invasive fibrosis scores as well as their optimal thresholds for MAFLD remains unknown. We validated the conventional non-invasive scores including aspartate aminotransferase to platelet ratio index, fibrosis-4 index, body mass index, aspartate aminotransferase/alanine aminotransferase ratio, diabetes score, and nonalcoholic fatty liver disease fibrosis score in patients with MAFLD. The results indicate that the conventional scores may lead to a high rate of misdiagnosis in MAFLD. A novel scoring system for fibrosis is urgently needed.

Citation: Wu YL, Kumar R, Wang MF, Singh M, Huang JF, Zhu YY, Lin S. Validation of conventional non-invasive fibrosis scoring systems in patients with metabolic associated fatty liver disease. World J Gastroenterol 2021; 27(34): 5753-5763

URL: https://www.wjgnet.com/1007-9327/full/v27/i34/5753.htm

DOI: https://dx.doi.org/10.3748/wjg.v27.i34.5753

\section{INTRODUCTION}

Nonalcoholic fatty liver disease (NAFLD), defined as excessive fat accumulation in liver cells in the absence of other liver diseases, has become a new epidemic due to its growing prevalence[1,2]. To date, NAFLD is believed to affect more than a quarter of the global population[2,3]. The natural history of NAFLD is highly variable; however, it is believed to progress through various fibrosis stages to end up in liver cirrhosis in a significant number of patients. The development and grade of liver fibrosis are strongly related with the adverse outcomes of NAFLD[4-6]. Thus, it is critical to identify patients with advanced fibrosis to optimize the management of NAFLD.

Liver biopsy is currently regarded as the "gold standard" for the diagnosis of liver fibrosis. However, due to the high prevalence of NAFLD, it is impossible to perform a biopsy for each patient. Moreover, the inherent issues including safety, sampling errors, and the inter- and intraobserver variation limit its application $[7,8]$. These limitations warrant the need for non-invasive scores for assessing liver fibrosis.

Numerous non-invasive assessment tools have been developed for diagnosis of advanced fibrosis[9]. The most widely used non-invasive scores include aspartate aminotransferase (AST) to platelet ratio index (APRI), fibrosis-4 index (FIB-4), body mass index (BMI), AST/alanine aminotransferase (ALT) ratio, diabetes score (BARD), and NAFLD fibrosis score (NFS). Most of them have been tested in subjects with NAFLD, showing great diagnostic accuracy in predicting fibrosis[10-13].

Metabolic associated fatty liver disease (MAFLD) is a recently proposed concept to replace NAFLD[14]. Significantly different from NAFLD, the MAFLD definition does not require the exclusion of any chronic liver diseases; however, the presence of metabolic associated disease or dysfunction is required[15,16]. It is known that metabolic profiles are associated with a risk for severe fibrosis in patients with NAFLD [17]. The MAFLD population has been found to have higher non-fibrosis scores than 
NAFLD individuals[18]. Thus, in the light of the new concept of MAFLD, which incorporates metabolic disorder, the performance of these non-invasive models requires re-evaluation and further validation. This study aimed to evaluate the utility of conventional non-invasive scoring systems in MAFLD.

\section{MATERIALS AND METHODS}

\section{Ethics}

The study protocol was approved by the Institutional Review Board of the First Affiliated Hospital of Fujian Medical University and was in accordance with the Declaration of Helsinki. Written consent forms were obtained from all patients. The data was anonymized prior to the analysis.

\section{Patients}

Consecutive patients with histologically confirmed MAFLD admitted to the First Affiliated Hospital of Fujian Medical University from 2005 to 2015 were retrospectively analyzed in this study.

\section{Histologic evaluation}

All patients enrolled in this study underwent percutaneous liver biopsy under ultrasonic guidance. The liver specimens were fixed in formalin, embedded in paraffin, and stained with hematoxylin and eosin and Masson's trichrome. The minimum biopsy length was $15 \mathrm{~mm}$, and at least six portal areas were required[19]. Histopathological slides were reviewed independently by two pathologists experienced in reading liver histopathology slides and were blinded to the patient's clinical data.

Fatty liver was defined as the presence of steatosis in at least $5 \%$ of hepatocytes. The liver fibrosis was graded as 0 to 4 points according to the Metavir fibrosis stage[20], where 0 = absence of fibrosis; 1 = perisinusoidal or periportal; 2 = perisinusoidal and portal/periportal; 3 = bridging fibrosis; 4 = cirrhosis. Advanced fibrosis was defined as stage 3 or 4 fibrosis (bridging fibrosis or cirrhosis).

\section{Diagnosis of MAFLD}

The diagnosis of MAFLD was based on the following criteria[15]: A histological evidence of hepatic steatosis and the presence of one of the following three conditions: $\mathrm{BMI} \geq 23 \mathrm{~kg} / \mathrm{m}^{2}$, presence of type 2 diabetes mellitus, or evidence of metabolic dysregulation. The metabolic dysregulation was defined by the presence of at least two of the following conditions: (1) Waist circumference $\geq 90 \mathrm{~cm}$ in men and $80 \mathrm{~cm}$ in women; (2) Blood pressure $\geq 130 / 85 \mathrm{mmHg}$ or specific drug treatment; (3) Plasma triglycerides $\geq$ $1.70 \mathrm{mmol} / \mathrm{L}$ or specific drug treatment; (4) Plasma high-density lipoprotein cholesterol $<1.0 \mathrm{mmol} / \mathrm{L}$ for men and $<1.3 \mathrm{mmol} / \mathrm{L}$ for women or specific drug treatment; (5) Prediabetes (i.e. fasting glucose levels 5.6-6.9 mmol/L or $2 \mathrm{~h}$ post-load glucose levels 7.8-11.0 mmol/L or glycated hemoglobin 5.7\%-6.4\%; (6) Homeostasis model assessment-insulin resistance score $\geq 2.5$; and (7) Plasma high-sensitivity Creactive protein level $>2 \mathrm{mg} / \mathrm{L}$.

According to the result of hepatitis B surface antigen (HBsAg) seropositivity, patients were divided into pure MAFLD group (HBsAg negative) and hepatitis B virus (HBV)-MAFLD group (HBsAg positive for $>6 \mathrm{mo}$ ) for the purpose of subgroup analysis.

\section{Demographic and laboratory evaluation}

The following data were collected at the time of biopsy from all patients: age, gender, BMI, waist circumference, history of diabetes, and hypertension. Laboratory parameters were as follows: Blood cell count, AST, ALT, y-glutamyl transpeptidase, albumin, globulin, bilirubin, fasting plasma glucose, total cholesterol, high-density lipoprotein cholesterol, low-density lipoprotein cholesterol, triglyceride, urea, creatinine, uric acid, fasting insulin, glycated hemoglobin, high-sensitivity C-reactive protein, and HBsAg.

All biochemical assessments were performed by standard laboratory methods. Noninvasive liver fibrosis assessment scores included APRI, FIB-4, BARD, and NFS, which were calculated based on previously published formulas as shown in Table 1[10,12,21, 22]. 
Table 1 An overview of formulas and cutoffs for determining non-invasive marker panels for detection of liver fibrosis[10-12,21,22]

\begin{tabular}{llll}
\hline Formula & Equation & Lower cutoff & Higher cutoff \\
\hline FIB-4 & $[$ Age $(\mathrm{yr}) \times \mathrm{AST}(\mathrm{IU} / \mathrm{L})] /$ platelet count $\left.\left(10^{9} / \mathrm{L}\right) \times[\mathrm{ALT}(\mathrm{IU} / \mathrm{L})]^{1 / 2}\right\}$ & 1.3 & $2.67[11]$ \\
& & 1.45 & $3.25[22]$ \\
APRI & {$\left[(\mathrm{AST} / \mathrm{ULN}) /\right.$ platelet count $\left.\left(10^{9} / \mathrm{L}\right)\right] \times 100$} & 0.5 & $1.5[21]$ \\
NFS & $-1.675+0.037 \times$ age $(\mathrm{yr})+0.094 \times$ BMI $\left(\mathrm{kg} / \mathrm{m}^{2}\right)+1.13 \times \mathrm{IFG} /$ diabetes $(\mathrm{yes}=1$, & -0.676 & $1.455[10]$ \\
& $\begin{array}{l}\text { no }=0)+0.99 \times \mathrm{AST} / \mathrm{ALT}-0.013 \times \text { platelet count }\left(\times 10^{9} / \mathrm{L}\right)-0.66 \times \text { albumin } \\
(\mathrm{g} / \mathrm{dL})\end{array}$ & & \\
BARD & $\begin{array}{l}\text { Scale } 0-4 ; \mathrm{BMI} \geq 28 \mathrm{~kg} / \mathrm{m}^{2}=1 \text { point; AST } / \mathrm{ALT} \geq 0.8=2 \text { points; Diabetes }=1 \\
\text { point }\end{array}$ & $2[12]$ & \\
\hline
\end{tabular}

ALT: Alanine aminotransferase; APRI: Aspartate aminotransferase to platelet ratio index; AST: Aspartate aminotransferase; BARD: Body mass index, aspartate aminotransferase/alanine aminotransferase ratio, diabetes score; BMI: Body mass index; FIB-4: Fibrosis-4 index; IFG: Impaired fasting glucose; NFS: Nonalcoholic fatty liver disease fibrosis score; ULN: Upper limit of normal.

\section{Statistical analysis}

Continuous variables are expressed as mean \pm SD or median (interquartile range) and were compared using Student's $t$ test in the case of normally distributed data or MannWhitney test in the remaining cases. Categorical variables are expressed as counts (percentages) and evaluated by $\chi^{2}$ test or the Fisher's exact test. The diagnostic accuracy of conventional non-invasive scoring systems was evaluated by the receiver operating characteristic (ROC) curve. The best cut-off values to determine the presence of advanced fibrosis were chosen based on Youden's index. The discrimination ability of the different models was compared using area under ROC curve (AUROC), positive predictive value (PPV), negative predictive value (NPV), sensitivity, specificity, and accuracy. Statistical analyses were performed using the SPSS software, version 18.0 (SPSS, Chicago, IL, United States) and MedCalc software version 15.2.2 (MedCalc Software, Mariakerke, Belgium). A $P$ value $<0.05$ was considered statistically significant.

\section{RESULTS}

\section{Baseline characteristics of the patients}

A total of 417 patients with biopsy-proven MAFLD were included in this study (Figure 1). All patients were treatment-naïve and did not receive nucleosides analogue treatment before biopsy. The mean age was $40.54 \pm 10.95$ years, and the BMI was 25.48 $\pm 2.66 \mathrm{~kg} / \mathrm{m}^{2}$. Of them, $354(84.9 \%)$ were male, $82(19.7 \%)$ had type 2 diabetes mellitus, $42(10.1 \%)$ had hypertension, and $156(37.4 \%)$ had advanced fibrosis (Table 2). Liver histological examination showed that $272(76.0 \%)$ HBV-MAFLD patients had significant inflammation (grade $\geq 2$ ). Antiviral therapy was initiated when chronic hepatitis B was diagnosed with the presence of significant inflammation (grade $\geq 2$ ) or significant liver fibrosis (stage $\geq 2$ ). Patients with advanced fibrosis were significantly older and had higher AST and lower albumin, triglyceride, and platelet counts. In addition, the FIB-4 $(P<0.001)$, NFS $(P<0.001)$, APRI $(P=0.003)$ and BARD $(P<0.001)$ scores were all significantly higher in patients with advanced fibrosis compared with patients with no/mild fibrosis.

\section{Performance of FIB-4, NFS, APRI, and BARD for advanced fibrosis in MAFLD}

The ROC curves were used to evaluate the utility of non-invasive scoring systems to identify advanced fibrosis (Figure 2). The AUROC was greatest for FIB-4 [0.736; 95\% confidence interval (CI): 0.691-0.778] followed by NFS (0.724; 95\%CI: 0.679-0.767), APRI (0.671; 95\%CI: 0.623-0.715), and BARD (0.609; 95\%CI: 0.560-0.656). The comparison between AUROCs showed that the discrimination abilities of FIB-4 and NFS were similar $(P=0.523)$. Similar results were also noted in NFS and APRI $(P=$ $0.080)$. The differences between FIB-4 and APRI $(P=0.001)$, FIB-4 and BARD $(P<$ $0.001)$, as well as NFS and BARD $(P<0.001)$ were statistically significant.

Table 3 summarizes the best cutoff values developed for prediction of advanced fibrosis by the four non-invasive models and the validation of previously reported cutoffs (for NAFLD) in this MAFLD cohort[10-12,21,22]. The best cutoff scores of FIB-4, 


\begin{tabular}{|c|c|c|c|c|}
\hline & Overall, $n=417$ & No/mild fibrosis, $n=261$ & Advanced fibrosis, $n=156$ & $P$ value \\
\hline Age (yr) & $40.54 \pm 10.95$ & $39.42 \pm 11.39$ & $42.41 \pm 9.94$ & 0.007 \\
\hline Male, $n(\%)$ & $354(84.9)$ & $219(83.9)$ & $135(86.5)$ & 0.468 \\
\hline BMI $\left(\mathrm{kg} / \mathrm{m}^{2}\right)$ & $25.48 \pm 2.66$ & $25.51 \pm 2.56$ & $25.42 \pm 2.83$ & 0.757 \\
\hline Diabetes mellitus, $n(\%)$ & $82(19.7)$ & $46(17.6)$ & $36(23.1)$ & 0.175 \\
\hline Hypertension, $n(\%)$ & $42(10.1)$ & 30 (11.5) & $12(7.7)$ & 0.212 \\
\hline $\operatorname{ALB}(g / L)$ & $42.53 \pm 4.96$ & $43.65 \pm 5.14$ & $40.65 \pm 3.98$ & $<0.001$ \\
\hline GLO $(\mathrm{g} / \mathrm{L})$ & $29.88 \pm 14.11$ & $29.11 \pm 6.00$ & $31.13 \pm 21.53$ & 0.160 \\
\hline $\operatorname{ALT}(\mathrm{U} / \mathrm{L})$ & $133.07 \pm 199.05$ & $126.93 \pm 151.37$ & $143.33 \pm 260.27$ & 0.416 \\
\hline AST (U/L) & $70.69 \pm 85.14$ & $63.24 \pm 62.19$ & $83.17 \pm 112.77$ & 0.021 \\
\hline GGT (U/L) & $89.64 \pm 109.58$ & $88.75 \pm 117.46$ & $91.11 \pm 95.28$ & 0.832 \\
\hline FPG (mmol/L) & $5.34 \pm 1.42$ & $5.28 \pm 1.20$ & $5.45 \pm 1.73$ & 0.251 \\
\hline $\mathrm{Cr}(\mu \mathrm{mol} / \mathrm{L})$ & $73.41 \pm 13.71$ & $74.00 \pm 13.50$ & $72.41 \pm 14.07$ & 0.273 \\
\hline $\mathrm{UA}(\mu \mathrm{mol} / \mathrm{L})$ & $368.49 \pm 82.08$ & $377.02 \pm 83.87$ & $353.77 \pm 76.98$ & 0.007 \\
\hline TG (mmol/L) & $1.67 \pm 1.12$ & $1.78 \pm 1.45$ & $1.48 \pm 0.71$ & 0.016 \\
\hline HDL-C (mmol/L) & $1.12 \pm 0.30$ & $1.12 \pm 0.29$ & $1.12 \pm 0.33$ & 0.959 \\
\hline $\operatorname{PLT}\left(\times 10^{9} / \mathrm{L}\right)$ & $204.00 \pm 64.22$ & $221.00 \pm 62.76$ & $175.52 \pm 56.22$ & $<0.001$ \\
\hline FIB-4 & $1.48 \pm 1.64$ & $1.19 \pm 1.65$ & $1.97 \pm 1.49$ & $<0.001$ \\
\hline APRI & $1.03 \pm 1.72$ & $0.84 \pm 1.33$ & $1.34 \pm 2.19$ & 0.003 \\
\hline NFS & $-2.16 \pm 1.38$ & $-2.56 \pm 1.24$ & $-1.50 \pm 1.33$ & $<0.001$ \\
\hline BARD (\%) & & & & $<0.001$ \\
\hline 0 & $209(50.1)$ & $145(55.6)$ & $64(41.0)$ & \\
\hline 1 & $86(20.6)$ & $59(22.6)$ & $27(17.3)$ & \\
\hline 2 & $92(22.1)$ & $48(18.4)$ & $44(28.2)$ & \\
\hline 3 & $25(6.0)$ & $8(3.1)$ & $17(10.9)$ & \\
\hline 4 & $5(1.2)$ & $1(0.4)$ & $4(2.6)$ & \\
\hline
\end{tabular}

ALB: Albumin; ALT: Alanine aminotransferase; APRI: Aspartate aminotransferase to platelet ratio index; AST: Aspartate aminotransferase; BARD: Body mass index, aspartate aminotransferase/alanine aminotransferase ratio, diabetes score; BMI: Body mass index; Cr: Creatinine; FIB-4: Fibrosis-4 index; FPG: Fasting plasma glucose; GGT: $\gamma$-Glutamyl transpeptidase; GLO: Globulin; HDL-C: High-density lipoprotein cholesterol; NFS: Non-alcoholic fatty liver disease fibrosis score; PLT: Platelet; TG: Triglyceride; UA: Uric acid.

APRI, NFS, and BARD for the diagnosis of advanced fibrosis in MAFLD were 1.05, $0.42,-2.1$, and 2, respectively. Most cutoff scores were lower than the prior reported thresholds for each model. Using the newly developed thresholds, the sensitivity, specificity, PPV, and NPV of the above four models ranged from $41.7 \%-81.4 \%, 44.4 \%$ $78.2 \%, 46.7 \%-55.8 \%$, and $69.2 \%-80.0 \%$, respectively (Table 3 ).

With the previously reported cutoff value of 1.30 (lower) and 2.67 (higher) of FIB-4 for advanced fibrosis, the PPV was only $57.3 \%$ and $69.2 \%$, and the NPV was $74.6 \%$ and $65.9 \%$, respectively. Similar results were found for the NFS score; the PPV and NPV of the well-accepted threshold (-1.455) were only $56.0 \%$ and $70.6 \%$ (Table 3).

The accuracy of the FIB- 4 and NFS scores, irrespective of the numerical value of cutoff used, was only $63.8 \%-68.8 \%$. The accuracy was even lower for APRI and BARD (58.3\%-64.5\%). On the other hand, 20\%-35\% patients with biopsy-proven advanced fibrosis were misdiagnosed as no advanced fibrosis by these four scores (Table 3).

Performance of the non-invasive scores in HBV-MAFLD and pure MAFLD subgroups According to the result of HBsAg, patients were divided into HBV-MAFLD (359, $86.1 \%)$ and pure MAFLD $(58,13.9 \%)$ subgroups. The difference in the baseline characteristics between HBV-MAFLD and pure MAFLD had been presented in our previous 
Table 3 Comparison of the diagnostic value among fibrosis-4 index, nonalcoholic fatty liver disease fibrosis score, aspartate aminotransferase to platelet ratio index, and body mass index, aspartate aminotransferase/alanine aminotransferase ratio, diabetes score in metabolic associated fatty liver disease

\begin{tabular}{|c|c|c|c|c|c|c|c|c|c|}
\hline & Cutoffs & AUROC & Accuracy, $\%$ & $\begin{array}{l}\text { Advanced } \\
\text { fibrosis being } \\
\text { missed, } \%^{1}\end{array}$ & Sensitivity, \% & Specificity, \% & PPV, $\%$ & NPV, $\%$ & $\begin{array}{l}\text { Youden } \\
\text { index }\end{array}$ \\
\hline \multirow[t]{5}{*}{ FIB-4 } & 1.05 & 0.736 & 66.2 & 20.3 & 73.7 & 61.7 & 53.5 & 79.7 & 0.354 \\
\hline & 1.30 & & 68.1 & 25.4 & 57.7 & 74.3 & 57.3 & 74.6 & 0.320 \\
\hline & 1.45 & & 68.8 & 26.5 & 52.6 & 78.5 & 59.4 & 73.5 & 0.311 \\
\hline & 2.67 & & 66.2 & 34.1 & 17.3 & 95.4 & 69.2 & 65.9 & 0.127 \\
\hline & 3.25 & & 66.7 & 34.4 & 14.1 & 98.1 & 81.5 & 65.6 & 0.122 \\
\hline \multirow[t]{3}{*}{ NFS } & -2.100 & 0.724 & 68.1 & 20.9 & 70.5 & 66.7 & 55.8 & 79.1 & 0.372 \\
\hline & -1.455 & & 66.2 & 29.5 & 44.9 & 78.9 & 56.0 & 70.6 & 0.238 \\
\hline & 0.676 & & 63.8 & 36.5 & 4.5 & 99.2 & 77.8 & 63.5 & 0.037 \\
\hline \multirow[t]{3}{*}{ APRI } & 0.42 & 0.671 & 58.3 & 20.0 & 81.4 & 44.4 & 46.7 & 80.0 & 0.258 \\
\hline & 0.50 & & 59.2 & 24.7 & 71.2 & 52.5 & 47.2 & 75.3 & 0.237 \\
\hline & 1.50 & & 63.3 & 34.6 & 22.4 & 87.7 & 52.2 & 65.4 & 0.101 \\
\hline BARD & 2 & 0.609 & 64.5 & 30.8 & 41.7 & 78.2 & 53.3 & 69.2 & 0.199 \\
\hline
\end{tabular}

Best cutoff value in bold.

${ }^{1}$ The percentage was calculated as the number of advanced fibrosis being missed diagnosed/the number of patients who were diagnosed as no advanced fibrosis by the non-invasive score $\times 100 \%$. APRI: Aspartate aminotransferase to platelet ratio index; AUROC: Area under the receiver operating characteristic curve; BARD: Body mass index, aspartate aminotransferase/alanine aminotransferase ratio, diabetes score; FIB-4: Fibrosis-4 index; NFS: Nonalcoholic fatty liver disease fibrosis score; NPV: Negative predictive value; PPV: Positive predictive value.

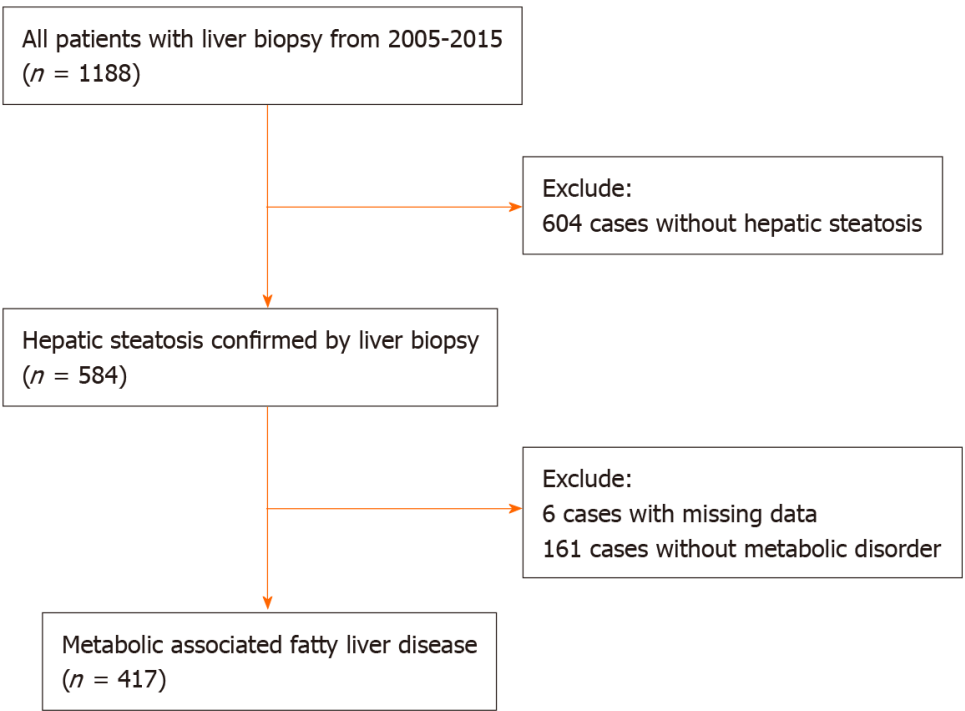

Figure 1 Flow chart of case selection.

work[23] and is shown in Supplementary Table 1. Briefly, the HBV-MAFLD group had higher ALT (135.82 $\pm 210.31 \mathrm{U} / \mathrm{L}$ vs $119.76 \pm 118.19 \mathrm{U} / \mathrm{L})$ and AST $(71.70 \pm 88.63 \mathrm{U} / \mathrm{L}$ vs $65.55 \pm 63.28 \mathrm{U} / \mathrm{L})$ levels and lower platelet $(198.59 \pm 60.33) \times 10^{\circ} / \mathrm{L}$ vs $(237.47 \pm$ $76.97) \times 10^{9} /$ L levels than the pure MAFLD group. The AUROCs of FIB-4, NFS, and APRI in the HBV-MAFLD group was $0.738,0.725$, and 0.671 , respectively, which were all higher than in the pure MAFLD group (FIB-4 0.658, NFS 0.692, and APRI 0.633). The AUROC of BARD was lower in the HBV-MAFLD group than in the pure MAFLD group (0.609 vs 0.644). Using different thresholds mentioned above, the overall performance of the FIB-4, APRI, and NFS, including sensitivity, specificity, PPV and 


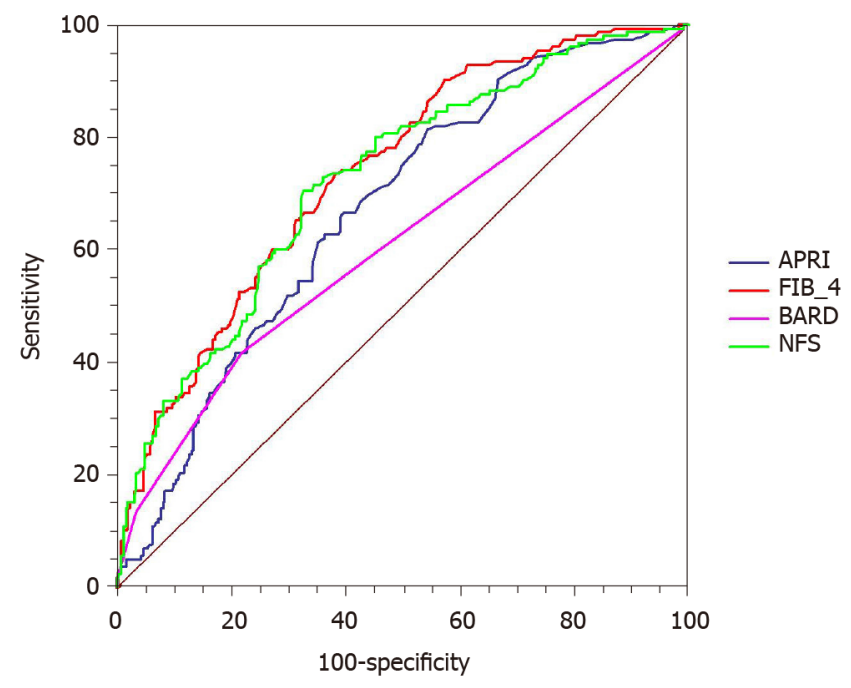

Figure 2 Receiver operating characteristic curves of different scores for advanced fibrosis. APRI: Aspartate aminotransferase to platelet ratio index; FIB-4: Fibrosis-4 index; NFS: Nonalcoholic fatty liver disease fibrosis score.

NPV, was better in the HBV-MAFLD group. The BARD score performed better in the pure MAFLD population (Table 4).

\section{DISCUSSION}

Non-invasive scoring systems are widely used to identify or exclude advanced fibrosis in patients with chronic liver disease. The main finding of our study is that FIB- 4 and NFS performed better than APRI and BARD in MAFLD patients. These scores are more useful in HBV-MAFLD than in pure MAFLD population. However, the performance of all above models was not as good as previously reported in NAFLD.

The non-invasive fibrosis scoring systems are derived from widely available clinical, laboratory, and anthropometric parameters. Although the APRI and BARD scores are more user-friendly, their discrimination ability in prediction of advanced fibrosis is not satisfactory in this group of MAFLD patients. The AUROC of BARD was 0.609, and the accuracy was less than $65 \%$. It is not a surprising result as the two variables for calculating BARD score (BMI and diabetes) were also variables for the diagnosis of MAFLD. This led to lower sensitivity and higher false positivity for detecting advanced fibrosis in MAFLD patients, thus the BARD score should not be recommended for MAFLD patients in clinical practice. Although APRI score was easy to calculate, it did not perform well in MAFLD as well. The NPV did not exceed $80 \%$, and the PPV was only around $50 \%$ at any cutoff value tested. Thus, APRI should not be used for assessment of advanced fibrosis in the MAFLD population either.

The calculations of FIB-4 and NFS require the use of more complex formulas and may not be easy to use. Even though the results of our study indicate that both FIB-4 and NFS significantly outperform APRI and BARD for the prediction of advanced fibrosis in MAFLD, the NPV and PPV of these two models did not reach the similar values reported by previous studies using the cohort of NAFLD patients[10,11,24]. According to the previously reported studies on patients with NAFLD $[10,11,24]$, when the cutoff of the FIB-4 and NFS was set at 1.3 and -1.455 , respectively, the NPV increased from $90 \%$ to $93 \%$. When the cutoff of the FIB- 4 was set at 2.67 , the PPV could reach $80 \%$. However, in our cohort of MAFLD patients, by using the aforementioned cutoffs, or the new threshold found in the present study, the NPV did not exceed $75 \%$, and PPV did not exceed $70 \%$. This finding is of utmost clinical importance and indicates that in the light of the new concept of MAFLD, newer non-invasive fibrosis scores need to be developed and validated to assess the presence of advanced fibrosis in patients with MAFLD. Another worrying yet important finding of our study is that the use of these non-invasive fibrosis scores, even with the new cutoffs, led to a misdiagnosis of no advanced fibrosis in $20 \%-35 \%$ of the patients in this cohort (Table 3) when the histology actually showed the presence of advanced fibrosis.

The occurrence of HBV infection and fatty liver disease is frequently seen in Asian countries[25]. As our cohort consisted of 359 (86.1\%) patients with chronic HBV 


\begin{tabular}{|c|c|c|c|c|c|c|c|c|c|c|c|c|c|}
\hline & \multirow[t]{2}{*}{ Cutoffs } & \multicolumn{2}{|c|}{ AUROC } & \multicolumn{2}{|c|}{ Accuracy, $\%$} & \multicolumn{2}{|c|}{ Sensitivity, \% } & \multicolumn{2}{|c|}{ Specificity, \% } & \multicolumn{2}{|c|}{ PPV, \% } & \multicolumn{2}{|c|}{ NPV, \% } \\
\hline & & A & B & A & B & A & B & A & B & A & B & A & B \\
\hline \multirow[t]{5}{*}{ FIB-4 } & 1.05 & 0.738 & 0.658 & 67.7 & 56.9 & 74.8 & 55.6 & 62.7 & 57.1 & 58.2 & 19.2 & 78.2 & 87.5 \\
\hline & 1.30 & & & 68.2 & 67.2 & 58.5 & 44.4 & 75.0 & 71.4 & 61.9 & 22.2 & 72.3 & 87.5 \\
\hline & 1.45 & & & 68.5 & 70.7 & 54.4 & 22.2 & 78.3 & 79.6 & 63.5 & 16.7 & 71.2 & 84.8 \\
\hline & 2.67 & & & 63.2 & 84.5 & 17.0 & 22.2 & 95.3 & 95.9 & 71.4 & 50.0 & 62.3 & 87.0 \\
\hline & 3.25 & & & 63.5 & 86.2 & 13.6 & 22.2 & 98.1 & 98.0 & 83.3 & 66.7 & 62.1 & 87.3 \\
\hline \multirow{3}{*}{ NFS } & -2.100 & 0.725 & 0.692 & 68.2 & 67.2 & 70.1 & 77.8 & 67.0 & 65.3 & 59.5 & 29.2 & 76.3 & 94.1 \\
\hline & -1.455 & & & 64.9 & 74.1 & 44.2 & 55.6 & 79.2 & 77.6 & 59.6 & 31.3 & 67.2 & 90.5 \\
\hline & 0.676 & & & 60.4 & 84.5 & 4.1 & 11.1 & 99.5 & 98.0 & 85.7 & 50.0 & 59.9 & 85.7 \\
\hline \multirow[t]{3}{*}{ APRI } & 0.42 & 0.671 & 0.633 & 59.3 & 51.7 & 81.6 & 77.8 & 43.9 & 46.9 & 50.2 & 21.2 & 77.5 & 92.0 \\
\hline & 0.50 & & & 60.2 & 55.2 & 70.7 & 77.8 & 52.8 & 51.0 & 51.0 & 22.6 & 72.3 & 92.6 \\
\hline & 1.50 & & & 61.0 & 77.6 & 23.1 & 11.1 & 87.3 & 89.8 & 55.7 & 16.7 & 62.1 & 84.6 \\
\hline BARD & 2 & 0.609 & 0.644 & 63.0 & 74.1 & 42.2 & 33.3 & 77.4 & 81.6 & 56.4 & 25.0 & 65.9 & 87.0 \\
\hline
\end{tabular}

APRI: Aspartate aminotransferase to platelet ratio index; AUROC: Area under the receiver operating characteristic curve; BARD: Body mass index, aspartate aminotransferase/alanine aminotransferase ratio, diabetes score; FIB-4: Fibrosis-4 index; NFS: Non-alcoholic fatty liver disease fibrosis score; NPV: Negative predictive value; PPV: Positive predictive value.

infection, we performed a subgroup analysis to test the performance of the noninvasive scores in a pure MAFLD group, which is very close to previous NAFLD and HBV-MAFLD group. Three of the four non-invasive models (FIB-4, NFS, and APRI) performed even worse in the pure MAFLD group than in the HBV-MAFLD group. We speculate that the higher levels of ALT and AST and lower platelets in HBV-MAFLD might result in higher scores of the non-invasive models and make them easier to discriminate the advanced fibrosis. As MAFLD is a new entity, this result further reinforces the need to develop and validate novel scoring systems for fibrosis in the MAFLD population.

The strength of our study is that, to our knowledge, this is the first validation of conventional non-invasive fibrosis scoring systems in a large sample of histologyproven MAFLD. However, this study has some limitations. Firstly, a large proportion of included patients $(86 \%)$ had concomitant chronic HBV infection, which could be a potential limitation as the western MAFLD population differs substantially from Asia's population in the HBsAg seropositivity rates, the results may only be applicable to a subset of the entire MAFLD pool namely HBV-MAFLD, which is the most important subtype of MAFLD in clinical practice in Asia. Secondly, as our study is a single-center study with only an Asian population, the findings will need further validation in other Asian and western cohorts.

\section{CONCLUSION}

APRI and BARD scores do not perform well and are not suitable for the diagnosis of advanced fibrosis in MAFLD. The FIB-4 and NFS could be more useful, and we propose a new threshold of 1.05 and -2.1, respectively, which had the best diagnostic performance for advanced fibrosis. There is an urgent need of a novel non-invasive scoring system for predicting advanced fibrosis in patients with MAFLD including its subtypes. 


\section{ARTICLE HIGHLIGHTS}

\section{Research background}

Metabolic associated fatty liver disease (MAFLD) is a new concept proposed in 2020. The clinical features of MAFLD would be different from nonalcoholic fatty liver disease.

\section{Research motivation}

Non-invasive fibrosis scores have been tested in subjects with nonalcoholic fatty liver disease showing great diagnostic accuracy in predicting fibrosis. But the utility of noninvasive fibrosis scores, as well as their optimal thresholds, needs re-evaluation in MAFLD.

\section{Research objectives}

This study aimed to evaluate the diagnostic performance of four non-invasive scores including aspartate aminotransferase to platelet ratio index (APRI), fibrosis-4 index (FIB-4), body mass index, aspartate aminotransferase/alanine aminotransferase ratio, and diabetes score (BARD), and nonalcoholic fatty liver disease fibrosis score (NFS) in patients with MAFLD.

\section{Research methods}

Consecutive patients with histologically-confirmed MAFLD admitted to a single medical center were included. The discrimination ability of different non-invasive scores was compared.

\section{Research results}

A total of 417 patients were included; $156(37.4 \%)$ of them had advanced fibrosis. The area under receiver operating characteristic curve of FIB-4, NFS, APRI, and BARD for predicting advanced fibrosis were $0.736,0.724,0.671$, and 0.609 , respectively. The area under receiver operating characteristic curve of FIB-4 and NFS was similar $(P=0.523)$, while the difference between FIB-4 and APRI $(P=0.001)$ and FIB-4 and BARD $(P<$ 0.001 ) was statistically significant. The best thresholds of FIB-4, NFS, APRI, and BARD for diagnosis of advanced fibrosis in MAFLD were 1.05, -2.1, 0.42, and 2, respectively. A subgroup analysis showed that FIB-4, APRI, and NFS performed worse in the pure MAFLD group than the HBV-MAFLD group.

\section{Research conclusions}

APRI and BARD scores do not perform well in MAFLD. The FIB-4 and NFS could be more useful, but a new threshold is needed.

\section{Research perspectives}

MAFLD is a new entity. The results of this study indicate the conventional scores may lead to misdiagnosis, and the development of novel scoring systems to assess fibrosis in the MAFLD population is urgently needed.

\section{REFERENCES}

1 Chalasani N, Younossi Z, Lavine JE, Charlton M, Cusi K, Rinella M, Harrison SA, Brunt EM, Sanyal AJ. The diagnosis and management of nonalcoholic fatty liver disease: Practice guidance from the American Association for the Study of Liver Diseases. Hepatology 2018; 67: 328-357 [PMID: 28714183 DOI: 10.1002/hep.29367]

2 Younossi Z, Anstee QM, Marietti M, Hardy T, Henry L, Eslam M, George J, Bugianesi E. Global burden of NAFLD and NASH: trends, predictions, risk factors and prevention. Nat Rev Gastroenterol Hepatol 2018; 15: 11-20 [PMID: 28930295 DOI: 10.1038/nrgastro.2017.109]

3 Sarin SK, Kumar M, Eslam M, George J, Al Mahtab M, Akbar SMF, Jia J, Tian Q, Aggarwal R, Muljono DH, Omata M, Ooka Y, Han KH, Lee HW, Jafri W, Butt AS, Chong CH, Lim SG, Pwu RF, Chen DS. Liver diseases in the Asia-Pacific region: a Lancet Gastroenterology \& Hepatology Commission. Lancet Gastroenterol Hepatol 2020; 5: 167-228 [PMID: 31852635 DOI: 10.1016/S2468-1253(19)30342-5]

4 Angulo P, Kleiner DE, Dam-Larsen S, Adams LA, Bjornsson ES, Charatcharoenwitthaya P, Mills PR, Keach JC, Lafferty HD, Stahler A, Haflidadottir S, Bendtsen F. Liver Fibrosis, but No Other Histologic Features, Is Associated With Long-term Outcomes of Patients With Nonalcoholic Fatty Liver Disease. Gastroenterology 2015; 149: 389-97.e10 [PMID: 25935633 DOI: 
10.1053/j.gastro.2015.04.043]

5 Hagström H, Nasr P, Ekstedt M, Hammar U, Stål P, Hultcrantz R, Kechagias S. Fibrosis stage but not NASH predicts mortality and time to development of severe liver disease in biopsy-proven NAFLD. J Hepatol 2017; 67: 1265-1273 [PMID: 28803953 DOI: 10.1016/j.jhep.2017.07.027]

6 Vilar-Gomez E, Calzadilla-Bertot L, Wai-Sun Wong V, Castellanos M, Aller-de la Fuente R, Metwally M, Eslam M, Gonzalez-Fabian L, Alvarez-Quiñones Sanz M, Conde-Martin AF, De Boer B, McLeod D, Hung Chan AW, Chalasani N, George J, Adams LA, Romero-Gomez M. Fibrosis Severity as a Determinant of Cause-Specific Mortality in Patients With Advanced Nonalcoholic Fatty Liver Disease: A Multi-National Cohort Study. Gastroenterology 2018; 155: 443-457.e17 [PMID: 29733831 DOI: 10.1053/j.gastro.2018.04.034]

7 Ratziu V, Charlotte F, Heurtier A, Gombert S, Giral P, Bruckert E, Grimaldi A, Capron F, Poynard T; LIDO Study Group. Sampling variability of liver biopsy in nonalcoholic fatty liver disease. Gastroenterology 2005; 128: 1898-1906 [PMID: 15940625 DOI: 10.1053/j.gastro.2005.03.084]

8 European Association for Study of Liver. Asociacion Latinoamericana para el Estudio del Higado. EASL-ALEH Clinical Practice Guidelines: Non-invasive tests for evaluation of liver disease severity and prognosis. J Hepatol 2015; 63: 237-264 [PMID: 25911335 DOI: 10.1016/j.jhep.2015.04.006]

9 Castera L, Friedrich-Rust M, Loomba R. Noninvasive Assessment of Liver Disease in Patients With Nonalcoholic Fatty Liver Disease. Gastroenterology 2019; 156: 1264-1281.e4 [PMID: 30660725 DOI: $10.1053 /$ j.gastro.2018.12.036]

10 Angulo P, Hui JM, Marchesini G, Bugianesi E, George J, Farrell GC, Enders F, Saksena S, Burt AD, Bida JP, Lindor K, Sanderson SO, Lenzi M, Adams LA, Kench J, Therneau TM, Day CP. The NAFLD fibrosis score: a noninvasive system that identifies liver fibrosis in patients with NAFLD. Hepatology 2007; 45: 846-854 [PMID: 17393509 DOI: 10.1002/hep.21496]

11 Shah AG, Lydecker A, Murray K, Tetri BN, Contos MJ, Sanyal AJ; Nash Clinical Research Network. Comparison of noninvasive markers of fibrosis in patients with nonalcoholic fatty liver disease. Clin Gastroenterol Hepatol 2009; 7: 1104-1112 [PMID: 19523535 DOI: 10.1016/j.cgh.2009.05.033]

12 Harrison SA, Oliver D, Arnold HL, Gogia S, Neuschwander-Tetri BA. Development and validation of a simple NAFLD clinical scoring system for identifying patients without advanced disease. Gut 2008; 57: 1441-1447 [PMID: 18390575 DOI: 10.1136/gut.2007.146019]

13 Kolhe KM, Amarapurkar A, Parikh P, Chaubal A, Chauhan S, Khairnar H, Walke S, Ingle M, Pandey V, Shukla A. Aspartate transaminase to platelet ratio index (APRI) but not FIB-5 or FIB-4 is accurate in ruling out significant fibrosis in patients with non-alcoholic fatty liver disease (NAFLD) in an urban slum-dwelling population. BMJ Open Gastroenterol 2019; 6: e000288 [PMID: 31275584 DOI: 10.1136/bmjgast-2019-000288]

14 Eslam M, Sanyal AJ, George J; International Consensus Panel. MAFLD: A Consensus-Driven Proposed Nomenclature for Metabolic Associated Fatty Liver Disease. Gastroenterology 2020; 158: 1999-2014.e1 [PMID: 32044314 DOI: 10.1053/j.gastro.2019.11.312]

15 Eslam M, Newsome PN, Sarin SK, Anstee QM, Targher G, Romero-Gomez M, Zelber-Sagi S, WaiSun Wong V, Dufour JF, Schattenberg JM, Kawaguchi T, Arrese M, Valenti L, Shiha G, Tiribelli C, Yki-Järvinen H, Fan JG, Grønbæk H, Yilmaz Y, Cortez-Pinto H, Oliveira CP, Bedossa P, Adams LA, Zheng MH, Fouad Y, Chan WK, Mendez-Sanchez N, Ahn SH, Castera L, Bugianesi E, Ratziu V, George J. A new definition for metabolic dysfunction-associated fatty liver disease: An international expert consensus statement. J Hepatol 2020; 73: 202-209 [PMID: 32278004 DOI: 10.1016/j.jhep.2020.03.039]

16 Fouad Y, Waked I, Bollipo S, Gomaa A, Ajlouni Y, Attia D. What's in a name? Liver Int 2020; 40: 1254-1261 [PMID: 32301554 DOI: 10.1111/liv.14478]

17 Petta S, Eslam M, Valenti L, Bugianesi E, Barbara M, Cammà C, Porzio M, Rosso C, Fargion S, George J, Craxì A. Metabolic syndrome and severity of fibrosis in nonalcoholic fatty liver disease: An age-dependent risk profiling study. Liver Int 2017; 37: 1389-1396 [PMID: 28235154 DOI: 10.1111/liv.13397]

18 Lin S, Huang J, Wang M, Kumar R, Liu Y, Liu S, Wu Y, Wang X, Zhu Y. Comparison of MAFLD and NAFLD diagnostic criteria in real world. Liver Int 2020; 40: 2082-2089 [PMID: 32478487 DOI: 10.111//liv.14548]

19 Bravo AA, Sheth SG, Chopra S. Liver biopsy. N Engl J Med 2001; 344: 495-500 [PMID: 11172192 DOI: 10.1056/nejm200102153440706]

20 Bedossa P, Poynard T. An algorithm for the grading of activity in chronic hepatitis C. The METAVIR Cooperative Study Group. Hepatology 1996; 24: 289-293 [PMID: 8690394 DOI: 10.1002/hep.510240201]

21 Wai CT, Greenson JK, Fontana RJ, Kalbfleisch JD, Marrero JA, Conjeevaram HS, Lok AS. A simple noninvasive index can predict both significant fibrosis and cirrhosis in patients with chronic hepatitis C. Hepatology 2003; 38: 518-526 [PMID: 12883497 DOI: 10.1053/jhep.2003.50346]

22 Sterling RK, Lissen E, Clumeck N, Sola R, Correa MC, Montaner J, S Sulkowski M, Torriani FJ, Dieterich DT, Thomas DL, Messinger D, Nelson M; APRICOT Clinical Investigators. Development of a simple noninvasive index to predict significant fibrosis in patients with HIV/HCV coinfection. Hepatology 2006; 43: 1317-1325 [PMID: 16729309 DOI: 10.1002/hep.21178]

23 Wang MF, Wan B, Wu YL, Huang JF, Zhu YY, Li YB. Clinic-pathological features of metabolic associated fatty liver disease with hepatitis B virus infection. World J Gastroenterol 2021; 27: 336344 [PMID: 33584066 DOI: 10.3748/wjg.v27.i4.336] 
24 McPherson S, Stewart SF, Henderson E, Burt AD, Day CP. Simple non-invasive fibrosis scoring systems can reliably exclude advanced fibrosis in patients with non-alcoholic fatty liver disease. Gut 2010; 59: 1265-1269 [PMID: 20801772 DOI: 10.1136/gut.2010.216077]

25 Polaris Observatory Collaborators. Global prevalence, treatment, and prevention of hepatitis B virus infection in 2016: a modelling study. Lancet Gastroenterol Hepatol 2018; 3: 383-403 [PMID: 29599078 DOI: 10.1016/S2468-1253(18)30056-6] 


\section{DS \\ Baishideng ${ }^{\circledR}$}

Published by Baishideng Publishing Group Inc

7041 Koll Center Parkway, Suite 160, Pleasanton, CA 94566, USA

Telephone: +1-925-3991568

E-mail: bpgoffice@wignet.com

Help Desk: https:/ /www.f6publishing.com/helpdesk

https://www.wjgnet.com

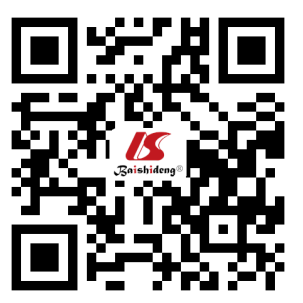

